> La prostaglandine D2 (PGD2) et ses dérivés sont des médiateurs lipidiques participant à l'homéostasie de la barrière épithéliale intestinale. Leur implication dans la physiopathologie des maladies inflammatoires chroniques de l'intestin reste encore débattue. Plusieurs résultats soulignent la dualité de la PGD2 quant à son rôle anti- ou pro-inflammatoire. Cette dualité semble liée à une expression différentielle de ses récepteurs par les cellules épithéliales intestinales et par les cellules immunocompétentes environnantes. Les cellules gliales du système nerveux entérique sont capables de sécréter ces médiateurs. Le rôle protecteur du système nerveux entérique dans le contrôle de l'homéostasie de la barrière épithéliale intestinale a été démontré. Ainsi, la PGD2 et ses dérivés se révèlent être de nouveaux acteurs de l'unité neuro-glio-épithéliale impliqués dans la régulation des fonctions des cellules épithéliales intestinales. <

L'implication des prostaglandines (PG) dans la physiopathologie des maladies inflammatoires chroniques de l'intestin (MICl) a été évoquée dès 1977 [1]. Selon les études et en fonction des médiateurs concernés, les rôles de ces molécules peuvent être protecteurs ou délétères. Ainsi, la prostaglandine ع2 (PGE2), issue de la voie des cyclo-oxygénases (COX), est classiquement décrite comme pro-inflammatoire et constitue le principal produit de cette voie métabolique présent sur le site de l'inflammation [2]. Dès 1978, des concentrations importantes de PGE2 ont été observées dans la muqueuse inflammatoire de patients atteints de rectocolite hémorragique, corrélées à la sévérité de la maladie [3]. L'impact délétère de cette production de PGE2 sur la barrière épithéliale intestinale a été en grande partie décrypté. II est lié à l'activation des récepteurs $E P 1$ et $\varepsilon P 4$ ( $\varepsilon$-prostanoid ( $\varepsilon P$ ) receptors 1 et 4$)^{1}$ de la PGE2,

${ }^{1}$ La famille des récepteurs de la PGE2 ( $\varepsilon$-prostanoid [EP] receptors) est subdivisée en 4 types: EPl à EP4.

\section{Prostaglandine D2 et homéostasie de la barrière épithéliale intestinale}

\section{Une relation équivoque}

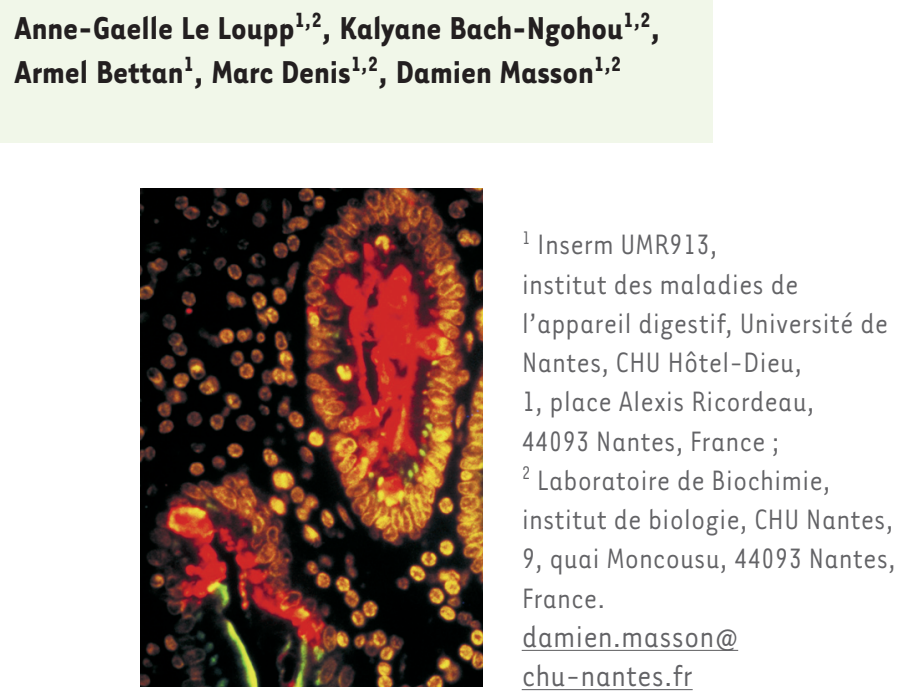

qui entraîne une rupture de l'intégrité de la barrière épithéliale intestinale [4]. À l'inverse de la PGE2, la prostaglandine D2 (PGD2), également issue de la voie des COX, serait plutôt anti-inflammatoire. Néanmoins, son rôle dans le contrôle de l'homéostasie de la barrière épithéliale intestinale n'est que partiellement élucidé. Les données de la littérature suggèrent qu'au cours des maladies inflammatoires chroniques de l'intestin, la production de PGD2 est plus tardive que celle de PGE2, mais qu'elle serait persistante et contemporaine de la résolution de certains processus inflammatoires [5-7]. Deux études récentes portant sur des patients atteints de rectocolite hémorragique montrent l'implication de ce médiateur lipidique dans la physiopathologie de ces maladies inflammatoires $[6,8]$.

\section{Une voie métabolique unique pour de multiples médiateurs lipidiques}

La PGD2 est un prostanoïde issu du métabolisme de l'acide arachidonique (AA), sous l'action couplée d'une cyclooxygénase (COX) et d'une prostaglandine D synthase (PGDS) (Figure I) [9, 10]. De nature instable, la PGD2 subit rapidement une déshydratation chimique non enzymatique, et se transforme en divers métabolites dont la 15-déoxy- $\Delta^{12,14}$ prostaglandine J2 (15d-PGJ2). Cette dernière joue un rôle sur la barrière épithéliale intestinale, notamment via son récepteur nucléaire PPAR $\gamma$ (peroxisome proliferator activated receptor $\gamma$ ). Ainsi, à partir d'un même précurseur, une voie métabolique identique permet d'aboutir à des composés lipidiques différents, dont les effets peuvent être multiples. 


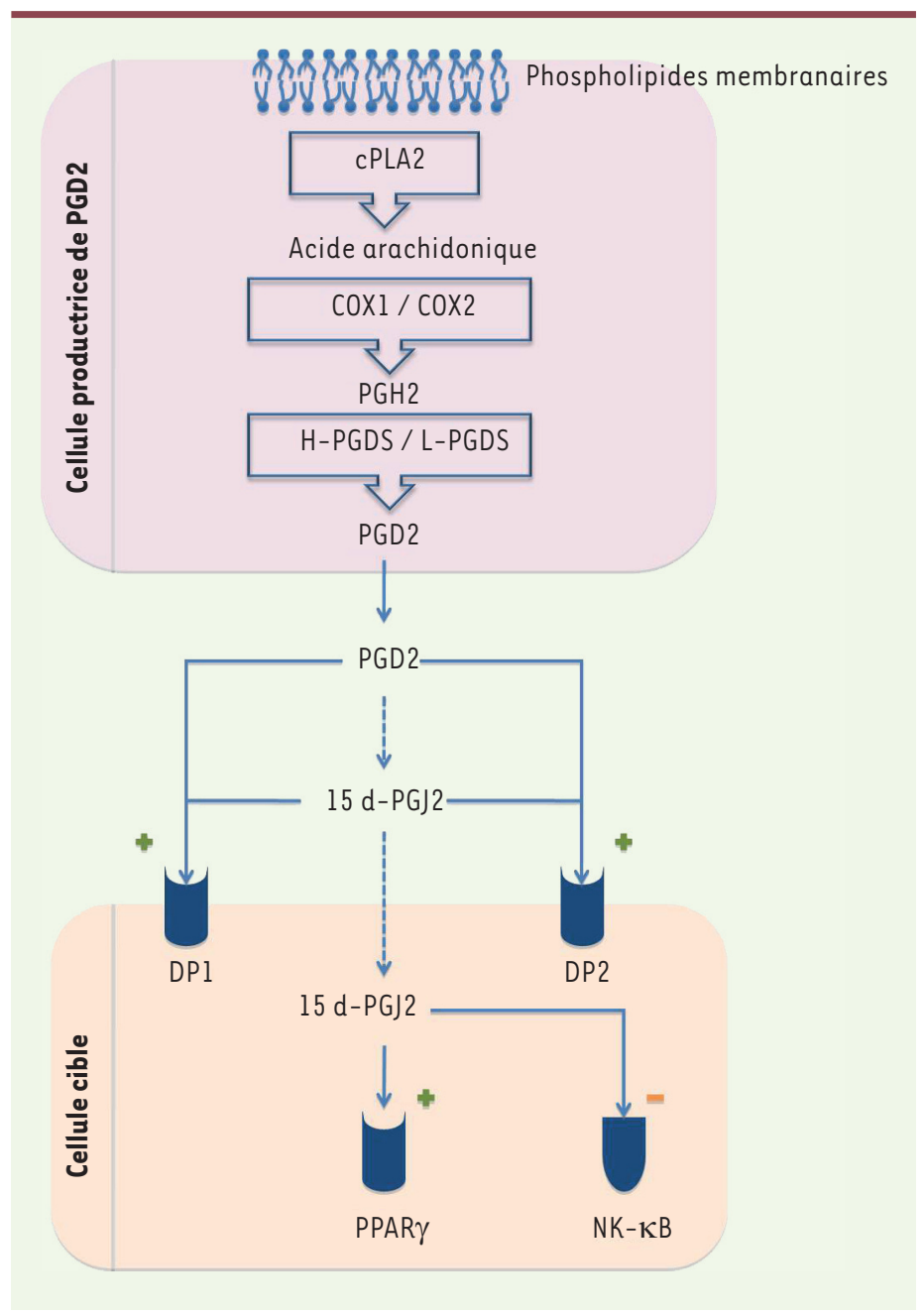

Figure 1. Voie métabolique de synthèse de la PGD2 et de la 15d-PGJ2.

Sous l'action de la phospholipase A2 (cPLA2), l'AA est libéré par hydrolyse des phospholipides membranaires. II est transformé en PGD2 grâce au couplage fonctionnel d'une COX à une PGDS. Une COX catalyse la réaction de bisoxygénation et d'hydroperoxydation de l'AA en prostaglandine G2 (PGG2), suivie d'une réduction en prostaglandine H2 (PGH2). La PGD2 est issue de l'isomérisation de la PGH2 par une PGDS. Deux cyclo-oxygénases sont décrites, COX1 et COX2 : alors que COX1 est exprimée de manière constitutive dans tous les tissus, COX2 est induite par de nombreux facteurs de croissance ou stimulus pro-inflammatoires. Ceux-ci induisent une redistribution généralement périnucléaire de COX2. C'est cette mobilité dynamique qui, en juxtaposant CPLA2, COX2 et les PG synthases, favorise l'induction de la synthèse des PG. II existe également deux types de prostaglandine $D$ synthase, différant par leur localisation tissulaire et leur fonctionnalité. La lipocaline-PGDS (LPGDS), indépendante du glutathion, a été initialement identifiée au niveau du système nerveux central [11]. Sa fonction est double : isomérase pour la transformation de la PGH2 en PGD2, et transporteur lipidique. En effet, la LPGDS est une glycoprotéine sécrétée dans les espaces extracellulaires qui a la capacité de lier et de transporter de petites molécules lipophiles. La PGDS hématopoïétique
(HPGDS) est, quant à elle, dépendante du glutathion, constitutive et de localisation cytosolique. Elle est décrite comme l'enzyme clé de la synthèse de la PGD2 par les cellules du système immunitaire et les mastocytes [12]. Au niveau intestinal, la HGPDS est principalement exprimée par les cellules épithéliales intestinales, les mastocytes et les fibroblastes [13-15]. L'expression de la LPGDS est plus restreinte, mais elle est induite en situation inflammatoire $[8,15]$. Cette prostaglandine $D$ synthase est préférentiellement exprimée par les cellules gliales du système nerveux entérique, en particulier au sein des ganglions des plexus sous muqueux et myentériques [15].

Les effets biologiques de la PGD2 sont relayés par l'activation de deux récepteurs à sept domaines transmembranaires : DP1 et DP2 (ou CRTH2, chemoattractant receptor-homologous molecule expressed on the Th2 cells) (Figure 1). L'activation de DPI couplé à la protéine Gs conduit à la production d'AMPc qui induit la voie de la protéine kinase $A$ ainsi qu'un flux calcique entrant [16]. DPl est impliqué dans la modulation des réponses immunitaires innée et adaptative [17], notamment via la réduction de l'infiltration leucocytaire et le contrôle de la synthèse des cytokines pro-inflammatoires [18]. À l'inverse, l'activation de DP2 couplé à la protéine Gi inhibe la production d'AMPc et induit une mobilisation intracellulaire de calcium [19]. DP2 est décrit en particulier pour son implication dans la promotion de la réaction allergique, le contrôle du recrutement de polynucléaires neutrophiles au niveau d'un site infectieux et de la production de cytokines pro- et anti-inflammatoires [20]. La 15d-PGJ2, par l'intermédiaire de PPAR $\gamma$, est impliquée dans le contrôle de la réponse inflammatoire et des processus de prolifération et de différenciation des cellules épithéliales intestinales (Figure 2) [15, 21].

\section{Dualité de la PGD2 dans la physiopathologie des maladies inflammatoires chroniques de l'intestin}

L'implication de la PGD2 dans la physiopathologie des maladies inflammatoires chroniques de l'intestin est actuellement débattue. Plusieurs arguments forts sont en faveur d'un impact bénéfique de la PGD2 lorsqu'elle interagit avec son récepteur DP1. Un modèle expérimental de colite chez le rat démontre que la PGD2 induit une diminution de l'infiltrat granulocytaire dans la muqueuse colique. Cet effet est également observé lors de l'administration d'un agoniste de DPl, suggérant l'implication de ce dernier [5]. Une étude basée sur l'analyse de biopsies coliques de patients présentant une rectocolite hémorragique montre l'implication de la PGD2 et de ce même récepteur DPI dans la résolution du processus inflammatoire et la persistance des rémissions au cours 
de cette maladie. En effet, une surexpression de DPl associée à une augmentation de la production de PGD2 est observée chez les patients en rémission par rapport aux patients en phase active de la maladie [6]. À l'inverse, un effet délétère de la PGD2 a été évoqué dans un modèle de colite induite par instillation d'acide trinitrobenzène sulphonique [22]. D'autres travaux évoquent l'implication de la voie COX/LPGDS dans la physiopathologie des maladies inflammatoires chroniques de l'intestin. L'étude d'Hokari et al., réalisée également sur des biopsies de patients présentant une rectocolite hémorragique, montre une augmentation de l'expression de la LPGDS, corrélée à la sévérité de la maladie [8]. Le phénotype des animaux chez lesquels une invalidation du gène codant pour la LPGDS a été réalisée ajoute à la complexité du rôle joué par la PGD2 dans le contrôle de l'homéostasie de la barrière épithéliale intestinale. Ainsi, chez les souris lpgds ${ }^{-/}$traitées par du dextran sulfate de sodium, l'atteinte inflammatoire est moins importante que celle observée chez des souris témoins également traitées par le dextran sulfate de sodium [7]. L'ensemble de ces données souligne toute la complexité du rôle de la PGD2 dans le contrôle de l'homéostasie de la barrière épithéliale intestinale et dans la physiopathologie des maladies inflammatoires chroniques de l'intestin. La dualité des effets observés repose probablement sur les différentes voies de signalisation activées par la PGD2 et la $15 \mathrm{~d}-\mathrm{PGJ} 2$, ainsi que sur la régulation de l'expression des récepteurs de ces molécules en situation inflammatoire. L'étude récente de Sturm et al. renforce cette hypothèse. Dans un modèle de colite induite chez le rat, les auteurs décrivent un rôle anti-inflammatoire de la PGD2 lorsqu'elle interagit avec DPl et, à l'inverse, un rôle pro-inflammatoire quand elle se lie à DP2. Ils observent également une expression différentielle de DP1 et DP2 sur les leucocytes du sang périphérique de patients présentant une rectocolite hémorragique active par rapport aux leucocytes de sujets contrôles [23]. Les aspects mécanistiques ne sont pas encore décryptés; en effet, les principales lignées cellulaires épithéliales utilisées dans les études, dérivées d'adénocarcinome colorectal, n'expriment ni DP1 ni DP2 [24], et, à l'heure actuelle, aucune étude du rôle de la PGD2 dans les maladies inflammatoires chroniques de l'intestin n'a été conduite sur des lignées de cellules épithéliales intestinales. L'action anti-inflammatoire de la PGD2 est en partie attribuée à son produit de déshydratation, la 15d-PGJ2. Ce dernier est un ligand naturel de PPAR $\gamma$, dont l'activation conduit à une inhibition de la transcription des cytokines pro-inflammatoires par les cellules immunocompétentes [25], à l'arrêt de la prolifération et à l'induction de la différenciation des cellules épithéliales intestinales (Figure 2) [15]. PPAR $\gamma$ est un acteur essentiel du maintien de l'immunité innée antimicrobienne au niveau du côlon [26]. De plus, il est clairement établi que la 15d-PGJ2 réprime l'activation de NF- $\kappa B$ en inhibant la phosphorylation de I $\kappa B$ par la kinase IKBK (Figure 1) [27, 28]. Enfin, une diminution de l'expression de PPAR $\gamma$ est observée en phase active de rectocolite hémorragique [29]. Un défaut d'expression de la voie de signalisation anti-inflammatoire PPAR $\gamma$ pourrait donc être l'un des éléments de la physiopathologie des maladies inflammatoires chroniques de l'intestin.

En ce qui concerne DPl, une voie de signalisation spécifique impliquée dans l'induction de l'expression de la mucine MUC5B a été décrite [30]. Une augmentation de l'expression des mucines MUC2 et MUC5AC, via DP1, a également été mise en évidence sur la lignée de cellules épithéliales intestinales LS174T, à caractère mucosécrétoire (Figure 2) [31]. Comme la fonction principale des mucines est de former un gel de mucus qui protège la muqueuse colique [28], le rôle bénéfique de la PGD2 dans les maladies inflammatoires chroniques de l'intestin pourrait être lié à l'augmentation de la sécrétion des mucines après activation de DPl. Les mucines MUC5AC et MUC2 sont en effet impliquées dans la réparation épithéliale au cours de ces maladies, via leur action sur la différenciation et la croissance cellulaire [32-34].

II est nécessaire de distinguer les effets directs de la PGD2 sur les cellules épithéliales intestinales par l'activation de ses récepteurs, des effets qu'elle exerce sur les cellules immunocompétentes environnantes, en particulier sur la régulation de leur recrutement. Dans un modèle de colite induite chez le rat par instillation d'acide trinitrobenzène sulphonique, l'administration de PGD2 inhibe l'infiltration des cellules granuleuses au niveau de la muqueuse, potentiellement par l'intermédiaire de son récepteur DPl, alors que l'administration d'un inhibiteur de cyclo-oxygénases induit cet infiltrat [5]. La participation de DP2 au recrutement des polynucléaires neutrophiles est également suggérée dans un modèle murin de sepsis sévère obtenu après ligature et perforation du cæcum [19]. Dans ce modèle, l'absence de DP2 (invalidation génique) est associée à une suppression de la production de cytokines pro-inflammatoires, à l'induction de la production d'interleukine (IL)-10 et à une augmentation du recrutement au niveau péritonéal des neutrophiles, via le récepteur de chimiokines CXCR2, dont l'activation induit la migration et l'activation de ces polynucléaires (Figure 2).

\section{Action paracrine de la PGD2 au sein de l'unité neuro-glio-épithéliale}

Plusieurs données permettent d'identifier l'origine de la PGD2 au niveau de la muqueuse intestinale. Le système nerveux entérique, composé de cellules gliales entériques et de neurones, s'intègre avec la barrière épithéliale intestinale au sein d'une unité fonctionnelle, appelée unité neuro-glio-épithéliale. Le fonctionnement de cette unité repose sur la proximité physique de ses différents composants [35]. De la même façon que les astrocytes du système nerveux central contrôlent la barrière hémato-encéphalique, les cellules gliales entériques sont des régulateurs majeurs des fonctions de la barrière épithéliale intestinale [36]. Ce contrôle est assuré par des médiateurs neuro-gliaux comme le TGF- $\beta 1$ (transforming growth factor $\beta 1$ ), le facteur VIP (vasoactive intestinal 


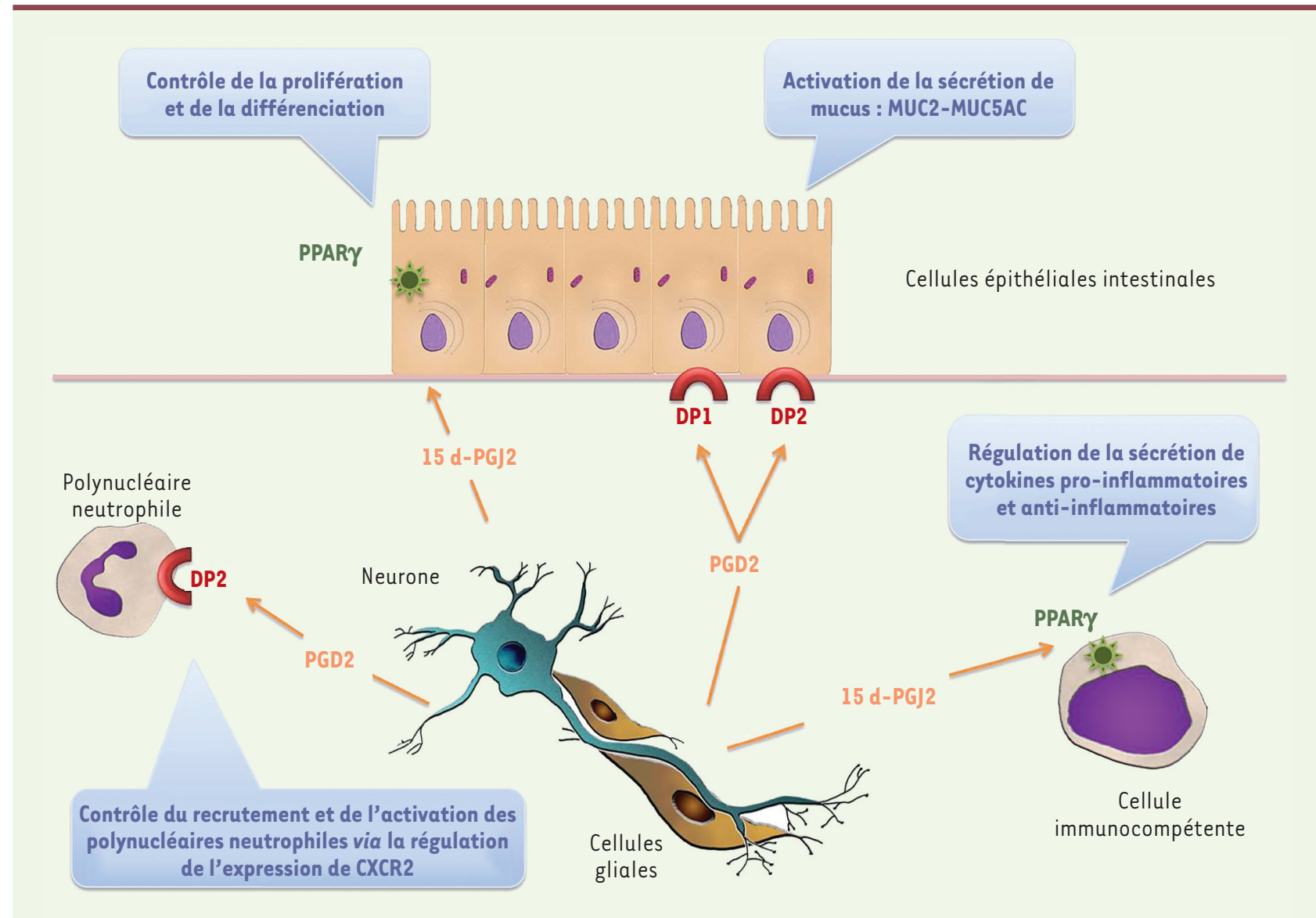

Figure 2. Schéma de synthèse du contrôle de l'homéostasie de la barrière épithéliale intestinale par la PGD2 et la $15 d-P G J 2$ sécrétées par les cellules gliales et les neurones du système nerveux entérique.

peptide) ou encore le GSNO (glutathion nitrosylé) [36-39]. La PGD2 semble également participer à cette relation glio-épithéliale. En effet, l'expression de la LPGDS par les cellules gliales du système nerveux entérique d'une part, et la modulation des mécanismes de réparation et de différenciation des cellules épithéliales intestinales par la $15 d-P G J 2$ d'autre part, confortent l'hypothèse selon laquelle le système nerveux entérique est à l'origine d'une production locale de PGD2 et de 15d-PGJ2 régulant les fonctions des cellules épithéliales intestinales (Figure 2) [15].

Ces données sont en accord avec le rôle joué par le système nerveux entérique dans la physiopathologie des maladies inflammatoires chroniques de l'intestin. Initialement considéré comme une cible de l'inflammation, du fait de l'observation de nombreuses lésions et modifications morphologiques, neurochimiques et fonctionnelles [40], le système nerveux entérique apparaît aujourd'hui comme un acteur essentiel du processus inflammatoire. En effet, les cellules gliales entériques expriment la machinerie cellulaire nécessaire à l'intégration d'un signal inflammatoire ayant pour origine les Toll-like receptors [41]. En réponse à ce signal, elles sont capables de proliférer et de sécréter des cytokines pro-inflammatoires comme l'IL- 6 ou l'IL-1 $\beta$ $[36,42]$, ou encore des prostanoïdes issus de la voie métabolique COX-LPGDS [15, 43].
En conclusion, de nombreux et récents travaux démontrent que la PGD2 et la $15 d-P G / 2$ jouent un rôle essentiel dans le contrôle et le maintien de l'homéostasie de la barrière épithéliale intestinale. Les cellules gliales du système nerveux entérique expriment les récepteurs et les enzymes indispensables à l'intégration d'un signal inflammatoire, ainsi qu'à l'établissement d'une relation paracrine avec les cellules épithéliales intestinales, notamment par la sécrétion des médiateurs lipidiques que sont la PGD2 et son dérivé, la 15d-PGJ2. Le rôle joué par ces médiateurs sur la barrière épithéliale intestinale reste complexe, en particulier en condition inflammatoire. Il fait intervenir une régulation de l'immunité innée et une régulation de la prolifération et de la différenciation des cellules épithéliales intestinales. $\diamond$

\section{SUMMARY}

Dual role for prostaglandin D2 in intestinal epithelial homeostasis

Prostaglandin D2 (PGD2) and derivatives are lipid mediators involved in the control of the intestinal epithelial barrier homeostasis. Their involvement in the pathophy- 
siology of chronic inflammatory bowel disease (IBD) is still debated. Several results highlight the duality of PGD2 as an anti- or pro-inflammatory mediator. This duality seems to be related to a differential expression of its receptors by intestinal epithelial cells and the surrounding immunocompetent cells. The enteric glial cells from the enteric nervous system (ENS) express the lipocalin-type-prostaglandin D synthase and secrete PGD2 and 15d-PGJ2. The protective role of the ENS in the homeostatic control of the epithelial intestinal barrier and its involvement in the pathogenesis of IBD have already been demonstrated. Thus, these lipid mediators seem to be new actors of the neuro-glio-epithelial unit and could play a crucial role maintaining gut barrier integrity. $\diamond$

\section{LIENS D'INTÉRÊT}

Les acteurs déclarent n'avoir aucun lien d'intérêt concernant les données publiées dans cet article.

\section{RéFÉRENCES}

1. Gould SR, Brash AR, Conolly ME. Increased prostaglandin production in ulcerative colitis. Lancet $1977 ; 2: 98$.

2. Murakami M, Naraba H, Tanioka T, et al. Regulation of prostaglandin $£ 2$ biosynthesis by inducible membrane-associated prostaglandin $\varepsilon 2$ synthase that acts in concert with cyclooxygenase-2.J Biol Chem $2000 ; 275$ : 32783-92.

3. Sharon P, Ligumsky M, Rachmilewitz D, Zor U. Role of prostaglandins in ulcerative colitis. Enhanced production during active disease and inhibition by sulfasalazine. Gastroenterology $1978 ; 75: 638-40$.

4. Rodriguez-Lagunas MJ, Martin-Venegas R, Moreno JJ, Ferrer R. PGE2 promotes Ca2+-mediated epithelial barrier disruption through $\mathrm{EPl}$ and $\varepsilon P 4$ receptors in Caco-2 cell monolayers. Am J Physiol Cell Physiol $2010 ; 299$ : C324-34.

5. Ajuebor MN, Singh A, Wallace JL. Cyclooxygenase-2-derived prostaglandin D(2) is an early antiinflammatory signal in experimental colitis. Am J Physiol Gastrointest Liver Physiol $2000 ; 279$ : G238-44.

6. Vong L, Ferraz JG, Panaccione R, et al. A pro-resolution mediator, prostaglandin D(2), is specifically up-regulated in individuals in long-term remission from ulcerative colitis. Proc Natl Acad Sci USA 2010 ; 107 : 12023-7.

7. Zamuner SR, Bak AW, Devchand PR, Wallace JL. Predisposition to colorectal cancer in rats with resolved colitis: role of cyclooxygenase-2-derived prostaglandin d2. Am J Pathol 2005; 167 : 1293-300.

8. Hokari R, Kurihara C, Nagata N, et al. Increased expression of lipocalin-type-prostaglandin D synthase in ulcerative colitis and exacerbating role in murine colitis. Am J Physiol Gastrointest Liver Physiol 2011 ; 300 : G401-8.

9. Smith WL, Urade Y, Jakobsson PJ. Enzymes of the cyclooxygenase pathways of prostanoid biosynthesis. Chem Rev $2011 ; 111: 5821-65$.

10. Malki S, Declosmenil F, Farhat A, et al. La prostaglandine D2 - Nouveaux rôles dans la gonade embryonnaire et pathologique. Med Sci (Paris) $2008 ; 24: 177-83$.

11. Urade $Y$, Fujimoto $N$, Hayaishi 0 . Purification and characterization of rat brain prostaglandin $D$ synthetase. J Biol Chem $1985 ; 260: 12410-5$.

12. Kanaoka Y, Fujimori K, Kikuno R, et al. Structure and chromosomal localization of human and mouse genes for hematopoietic prostaglandin $D$ synthase. Conservation of the ancestral genomic structure of sigma-class glutathione S-transferase. EurJ Biochem $2000 ; 267: 3315-22$.

13. Hokari R, Nagata N, Kurihara C, et al. Increased expression and cellular localization of lipocalin-type prostaglandin D synthase in Helicobacter pylori-induced gastritis. J Pathol $2009 ; 219: 417-26$.

14. Park JM, Kanaoka $Y$, Eguchi $N$, et al. Hematopoietic prostaglandin $D$ synthase suppresses intestinal adenomas in ApcMin/+ mice. Cancer Res $2007 ; 67: 881-9$

15. Bach-Ngohou K, Mahe MM, Aubert P, et al. Enteric glia modulate epithelial cell proliferation and differentiation through 15-deoxy-12,14-prostaglandin J2.J Physiol $2010 ; 588: 2533-44$.

16. Boie Y, Sawyer N, Slipetz DM, et al. Molecular cloning and characterization of the human prostanoid DP receptor. J Biol Chem 1995 ; 270 : 18910-6.

17. Hammad H, Kool M, Soullie T, et al. Activation of the D prostanoid 1 receptor suppresses asthma by modulation of lung dendritic cell function and induction of regulatory T cells. J Exp Med 2007 ; $204: 357-67$.

18. Rajakariar R, Hilliard M, Lawrence T, et al. Hematopoietic prostaglandin D2 synthase controls the onset and resolution of acute inflammation through PGD2 and 15-deoxyDelta12 14 PGJ2. Proc Natl Acad Sci USA 2007 ; 104 : 20979-84.

19. Ishii M, Asano K, Namkoong H, et al. CRTH2 is a critical regulator of neutrophil migration and resistance to polymicrobial sepsis. J Immunol 2012 ; 188 : 5655-64.

20. Satoh T, Moroi R, Aritake K, et al. Prostaglandin D2 plays an essential role in chronic allergic inflammation of the skin via CRTH2 receptor. J Immunol 2006 ; 177 : 2621-9.
21. Ricote M, Li AC, Willson TM, et al. The peroxisome proliferator-activated receptor-gamma is a negative regulator of macrophage activation. Nature $1998 ; 391: 79-82$.

22. Zamuner SR, Warrier N, Buret AG, et al. Cyclooxygenase 2 mediates postinflammatory colonic secretory and barrier dysfunction. Gut $2003 ; 52$ : 1714-20.

23. Sturm EM, Radnai B, Jandl K, et al. Opposing roles of prostaglandin D2 receptors in ulcerative colitis. J Immunol $2014 ; 193: 827-39$.

24. Hawcroft G, Gardner SH, Hull MA. Expression of prostaglandin D2 receptors DP1 and DP2 by human colorectal cancer cells. Cancer Lett $2004 ; 210$ : 81-4.

25. Jiang C, Ting AT, Seed B. PPAR-gamma agonists inhibit production of monocyte inflammatory cytokines. Nature $1998 ; 391: 82-6$.

26. Peyrin-Biroulet L, Beisner J, Wang G, et al. Peroxisome proliferatoractivated receptor gamma activation is required for maintenance of innate antimicrobial immunity in the colon. Proc Natl Acad Sci USA 2010 ; 107 : 8772-7.

27. Castrillo A, Diaz-Guerra MJ, Hortelano S, et al. Inhibition of IkappaB kinase and IkappaB phosphorylation by 15 -deoxy-Delta $(12,14)$-prostaglandin J(2) in activated murine macrophages. Mol Cell Biol $2000 ; 20: 1692-8$.

28. Hansson GC. Role of mucus layers in gut infection and inflammation. Curr Opin Microbiol 2012 ; 15 : 57-62.

29. Dubuquoy L, Jansson $E A$, Deeb $S$, et al. Impaired expression of peroxisome proliferator-activated receptor gamma in ulcerative colitis. Gastroenterology 2003 ; 124 : 1265-76.

30. Choi $Y H$, Lee SN, Aoyagi H, et al. The extracellular signal-regulated kinase mitogen-activated protein kinase/ribosomal $\$ 6$ protein kinase 1 cascade phosphorylates CAMP response element-binding protein to induce MUC5B gene expression via D-prostanoid receptor signaling. J Biol Chem 2011; 286 : 34199-214.

31. Wright DH, Ford-Hutchinson AW, Chadee K, Metters KM. The human prostanoid DP receptor stimulates mucin secretion in LS174T cells. $\mathrm{Br}$ J Pharmacol 2000 ; 131 : 1537-45.

32. Velcich $A$, Yang $W$, Heyer J, et al. Colorectal cancer in mice genetically deficient in the mucin Muc2. Science 2002 ; 295 : 1726-9.

33. Buisine MP, Desreumaux P, Leteurtre $\varepsilon$, et al. Mucin gene expression in intestinal epithelial cells in Crohn's disease. Gut 2001 ; 49 : 544-51.

34. Zouiten-Mekki L, Serghini M, Fekih M, et al. Rôle de la cellule épithéliale dans l'homéostasie intestinale et les maladies inflammatoires chroniques de l'intestin. Med Sci (Paris) 2013 ; 29 : 1145-50.

35. Neunlist M, Van Landeghem L, Bourreille A, Savidge T. Neuro-glial crosstalk in inflammatory bowel disease. J Intern Med 2008 ; $263: 577-83$.

36. Savidge TC, Newman P, Pothoulakis C, et al. Enteric glia regulate intestinal barrier function and inflammation via release of S-nitrosoglutathione. Gastroenterology 2007 ; 132 : 1344-58.

37. Neunlist M, Van Landeghem L, Mahe MM, et al. The digestive neuronal-glialepithelial unit: a new actor in gut health and disease. Nat Rev Gastroenterol Hepatol $2013 ; 10: 90-100$.

38. Neunlist M, Toumi F, Oreschkova T, et al. Human ENS regulates the intestina epithelial barrier permeability and a tight junction-associated protein Z0-1 via VIPergic pathways. Am J Physiol Gastrointest Liver Physiol 2003 ; 285 : G1028-36.

39. Neunlist M, Aubert P, Bonnaud S, et al. Enteric glia inhibit intestinal epithelial cell proliferation partly through a TGF-betal-dependent pathway. Am J Physiol Gastrointest Liver Physiol 2007 ; 292 : G231-41.

40. Villanacci V, Bassotti G, Nascimbeni R, et al. Enteric nervous system abnormalities in inflammatory bowel diseases. Neurogastroenterol Motil $2008 ; 20: 1009-16$.

41. Barajon I, Serrao G, Arnaboldi F, et al. Toll-like receptors 3, 4, and 7 are expressed in the enteric nervous system and dorsal root ganglia.J Histochem Cytochem 2009; 57 : 1013-23.

42. Ruhl A. Glial cells in the gut. Neurogastroenterol Motil $2005 ; 17: 777-90$.

43. Mosnier JF, Jarry A, Camdessanche JP, Antoine JC, Laboisse CL. In situ evidence of involvement of Schwann cells in ulcerative colitis: autocrine and paracrine signaling by A disintegrin and metalloprotease-17-mediated tumor necrosis factor alpha production. Hum Pathol 2009 ; 40 : 1159-67. 